\title{
Dairesel Sezgisel Bulanık Çok Kriterli Karar Verme Metodolojisi
}

\author{
Esra Çakır ${ }^{1}$, Mehmet Ali Taş ${ }^{*}$ \\ ${ }^{1}$ Galatasaray Üniversitesi, Mühendislik ve Teknoloji Fakültesik, Endüstri Mühendisliği, İstanbul, Türkiye, (ORCID: 0000-0003-4134-7679), ecakir@gsu.edu.tr \\ ${ }^{2 *}$ Türk-Alman Üniversitesi, Mühendislik Fakültesi, Endüstri Mühendisliği, İstanbul, Türkiye, (ORCID: 0000-0003-3333-7972), mehmetali.tas@tau.edu.tr
}

(1st International Conference on Applied Engineering and Natural Sciences ICAENS 2021, November 1-3, 2021)

(DOI: $10.31590 /$ ejosat.1011888)

\begin{abstract}
ATIF/REFERENCE: Çakır, E. \& Taş, M. A. (2021). Dairesel Sezgisel Bulanık Çok Kriterli Karar Verme Metodolojisi. Avrupa Bilim ve Teknoloji Dergisi, (28), 900-905.

$\ddot{O} z$

Sağlık turizmi günümüzün hızla büyüyen ekonomik alanlarından biridir. Sağlık turizmininden elde edilecek gelirleri arttırmak için tanıtım çalışmaları yürütülmelidir. Bu çalışmaların etkili bir şekilde yapılması için en iyi merkezin seçilmesi gereklidir. En iyi sağlık turizmi merkezi seçiminde çok kriterli karar verme yaklaşımlarından faydalanılabilir. Bu çalışmada, Türkiye'deki dört alternatif sağlık turizmi merkezinin değerlendirilmesi için bir bulanık çok kriterli karar verme yöntemi önerilmektedir. Alternatifler, uzmanlar tarafından sezgisel bulanık sayılarla değerlendirilerek yeni bir dairesel sezgisel bulanık çok kriterli karar verme yaklaşımı geliştirilmiştir. Mevcut literatüre ek olarak, bu çalışma yeni durulaştırma fonksiyonları önererek dairesel sezgisel bulanık kümelere katkıda bulunmaktadır. Uygulamanın, gelecekteki dairesel sezgisel bulanık çok kriterli karar verme yöntemlerinin uygulanmasına rehberlik etmesi amaçlamaktadır.
\end{abstract}

Anahtar Kelimeler: Bulanık Mantık, Dairesel Sezgisel Bulanık Küme, Çok Kriterli Karar Verme, Sağlık Turizmi.

\section{Circular Intuitionistic Fuzzy Multi-Criteria Decision Making Methodology}

\begin{abstract}
Health tourism is one of today's rapidly growing economic areas. Promotional activities must be carried out in order to increase the income to be obtained from health tourism. It is necessary to select the best center for these studies to be prepared effectively. Multicriteria decision-making approaches can be considered in use of the selection of the best health tourism center. This study proposes a fuzzy multi criteria decision making methodology in evaluation of four alternative health tourism centers in Turkey. A new circular intuitionistic fuzzy multi-criteria decision-making approach was developed by evaluating the alternatives with intuitionistic fuzzy numbers by experts. In addition to the existing literature, this study contributes to circular intuitionistic fuzzy sets by proposing new defuzzification functions. The study aims to guide future circular intuitionistic fuzzy multi-criteria decision making approaches.
\end{abstract}

Keywords: Fuzzy Logic, Circular Intuitionistic Fuzzy Sets, Multi-Criteria Decision Making, Health Tourism.

\footnotetext{
* Corresponding Author: mehmetali.tas@tau.edu.tr
} 


\section{Giriş}

Sağlık için seyahat etmek bilinen en eski turizm nedenlerinden biridir (Tureac \& Turtureanu, 2010). İlk tanımlamalardan birini yapan Uluslararası Resmi Seyahat Örgütleri Birliği'ne göre sağlık turizmi "kaplıca alanları ve eşsiz iklim alanları başta olmak üzere doğal kaynaklara sahip kırsal alanlarda sağlık tesislerinin sağlanması" olarak ifade edilir (Lee \& Li, 2019). İlk zamanlarda, sağlık turizmi ile tıbbi tedavi ve şifa için seyahat etmek gibi sınırlı bir anlam işaret edilmiştir (Yang vd., 2014). Zamanla bu anlam genişletilerek daha kapsayıcı bir hal almıştır. Güncel bir örnek olarak ise, sağlık turizmi için “...birincil motivasyonu bireylerin kendi ihtiyaçlarını karşılama kapasitelerini artıran ve çevrelerinde ve toplumda birey olarak daha iyi işlev gören tıbbi ve sağlıklı yaşam temelli faaliyetler yoluyla fiziksel, zihinsel ve/veya ruhsal sağlığa katkı sağlayan turizm" tanımlaması verilebilir (UNWTO, 2018). Sağlık (health) turizminin yerine sıklıkla medikal (medical) turizm ya da hastane (hospital) turizmi gibi kavramlar kullanılmaktadır (Kušen, 2011; Amouzagar vd., 2016). Aslında, sağlik turizmi diğer tüm tanımlamaları da kapsayan bir konsept olarak değerlendirilebilir (Carrera \& Bridges, 2006; Izadi vd., 2012). Sağlık turizmi medikal turizm, engelli turizmi, yaşlı turizmi ve termal/spa turizmi gibi alt türlere ayırmak mümkündür (Kantar \& Işık, 2014).

Turizm, yarattığı ekonomi ve yeni istihdam firsatları ile dünyanın en büyük endüstrilerinden biri haline gelmiştir (Sariisik vd., 2011). 2018 yılındaki istatistiklere göre Türkiye, yaklaşık 46 milyon ile tüm dünyada en çok turistin geldiği on ülke içinde altıncı sırada yer almaktadır (UNWTO, 2019). 2003 ile 2020 arasındaki dönemde en yüksek turizm gelirinin elde edildiği 2019 yılında Türkiye'ye 34,520 milyar dolar girişi olmuştur. Bu turizm gelirinin aynı yıldaki gayri safi yurt içi hasıla (GSYİH) içindeki payı \%4,6 olarak gerçekleşmiştir (TÜRSAB, 2021). Ekonomik kalkınma için sağlık turizmi gelirlerini arttrmak yardımcı bir etken olabilir sunar (Akinboade \& Braimoh, 2010). Bu amaçla Türkiye Kültür ve Turizm Bakanlığı, Türkiye'nin dünya sağlık turizmi pazarındaki payını arttırmak için kısa, orta ve uzun vadeli stratejiler belirlemiştir (T. C. Kültür ve Turizm Bakanlığı, 2021). Stratejiler oluşturulurken sağlık turizmi merkezlerini değerlendirmek gerekir. Sağlık turizmi merkezlerinin değerlendirilmesinde çok sayıda kriter göz önünde bulundurulmalıdır (Çakır \& Ulukan, 2020). Bu nedenle bu problemlerin çözümünde çok kriterli karar verme yöntemleri kullanılabilir (Ghasemi vd., 2021). Literatürde daha önce Hung vd. (2014) medikal turizm servislerinin sıralanmasında DANP ve VIKOR yöntemlerini birlikte kullanmıştır. Roy vd. (2018), Hindistan'daki şehirleri medikal turizm yönünden sıralamada RAHP (rough AHP) ve MABAC kullanmıştır. Pan vd. (2019) ise Çin'deki sağllk turizmi bölgelerinin değerlendirilmesinde çok kriterli karar verme yöntemi olarak ANP'den faydalanmıştır. Ghasemi vd. (2021) sürdürülebilir sağlik turizmi destinasyonlarını incelemede bulanık SWARA ve PROMETHEE yöntemlerini kullanmıştır. Görüldüğü gibi, çok kriterli karar verme yöntemlerinin uygulanmasında uzman görüşlerini yansıtabilmek için sıklıkla bulanık mantık ve bulanık küme teorisinden faydalanılmaktadır.

Bulanık küme teorisi, belirsizliği ifade etmek için Zadeh tarafından 1965 yılında ortaya konmuştur (Zadeh, 1965). Yıllar içinde bu teorinin temel alındığı nötrozofik bulanık kümeler (Smarandache, 1998), tereddütlü bulanık kümeler (Torra, 2010), Pisagor bulanık kümeler (Yager, 2013) ve küresel bulanık sayılar (Kutlu Gündoğdu \& Kahraman, 2019) gibi çok sayıda bulanık küme uzantısı önerilmiştir. Bunlardan biri de literatürde yaygın olarak kullanılanılan ve Atanassov (1986) tarafindan tanitılan sezgisel bulanık kümelerdir (SBK). Devamında yine Atanassov, bu konsepti geliştirerek 2020'de dairesel sezgisel bulanık kümeler (D-SBK) olarak adlandırılan yeni bir SBK uzantısı önermiştir (Atanassov, 2020). Bu yeni kümenin geliştirildiği SBK'den merkezi üye olma ve üye olmama derecelerinden oluşan bir yarıçap notasyonu içermesi ile farklılaşır (Kahraman \& Alkan, 2021).

$\mathrm{Bu}$ çalışmada, Türkiye'deki sağlık turizmi merkezlerinin değerlendirilmesi ve en iyi alternatifin belirlenmesi yer almaktadır. Böylece sağlık turizmindeki değerlendirme problemlerinin çözümüne katkı sunulması amaçlanmaktadır. Çalışmanın bir diğer önemli katkısı, dairesel SBK için durulaştırma fonksiyonlarının tanıtılmasıdır. Çok kriterli karar verme yöntemlerinde dairesel sezgisel bulanık kümelerin kullanımının yaygınlaştırılması istenmektedir. $\mathrm{Bu}$ çalışmanın bahsi geçen alanlarda literatürdeki boşlukları doldurduğu ve literatüre katkıda bulunduğu düşünülmektedir.

Makalenin geri kalanı şu şekilde düzenlenmiştir. Bölüm 2, yeni tanımlanmış durulaştırma fonksiyonları ile dairesel sezgisel bulanık kümeleri (D-SBK) tanıtmaktadır. Bölüm 3'te, dairesel sezgisel bulanık çok kriterli karar verme (D-SB ÇKKV) metodolojisinin uygulama adımları verilmiştir. Bölüm 4'te sağlık turizmi merkezi seçiminde önerilen yaklaşım uygulanmıştır. Son bölümde ise vaka çalışmasının sonuçları ve gelecek çalışmalar için öneriler yer almaktadır.

\section{Dairesel Sezgisel Bulanık Küme}

Bu bölümde dairesel sezgisel bulanık kümenin (D-SBK) ön bilgileri ve tanımları verilmektedir.

Tanım 1: (Atanassov, 1986) $X=\left\{x_{1}, x_{2}, \ldots, x_{n}\right\}$ siradan sabitlenmiş bir küme olsun, $X$ 'te bir SB sayı olan $A$ şu şekilde verilir:

$$
A=\left\{<x, u_{A}(x), v_{A}(x)>x \in X\right\}
$$

öyle ki " $u_{A}: X \rightarrow[0,1]$ " ve " $v_{A}: X \rightarrow[0,1]$ "; $0 \leq u_{A}(x)+$ $v_{A}(x) \leq 1, \forall x \in X$ koşulunu sağlar. Bu sayılar, $A$ kümesindeki $x$ elemanının üye olma derecesini " $u_{A}(x)$ " ve üye olmama derecesini " $v_{A}(x)$ " göstermektedir.

$x$, X'te bir eleman olmak üzere, " $<u_{A}(x), v_{A}(x)>$ " çifti sezgisel bulanık sayı (SBS) olarak adlandırılır (Chen \& Tan, 1994). Daha kolay bir gösterimle, $u_{\tilde{a}} \in[0,1], v_{\tilde{a}} \in[0,1]$ ve $0 \leq u_{\tilde{a}}+v_{\tilde{a}} \leq 1$ koşulları ile $\tilde{a}=<u_{\tilde{a}}, v_{\tilde{a}}>$ şeklinde de ifade edilebilir. Belirsizlik derecesi $\pi_{\tilde{a}}, \pi_{\tilde{a}} \in[0,1]$ ve $\pi_{\tilde{a}}=1-u_{\tilde{a}}-$ $v_{\tilde{a}}$ koşullarını sağlar.

Tanım 2: $\tilde{a}=\left(u_{\tilde{a}}, v_{\tilde{a}}\right)$ bir SB sayı olsun. Bu sayı üzerine tanımlanan puan fonksiyonu (durulaştırma fonksiyonu) $\mathrm{S}$ ve doğruluk fonksiyonu aşağıdaki gibi tanımlanır (Atanassov, 2020):

$$
\begin{aligned}
& S(\tilde{a})=u_{\tilde{a}}-v_{\tilde{a}} \quad \text { için } S(\tilde{a}) \in[-1,1] \\
& H(\tilde{a})=u_{\tilde{a}}+v_{\tilde{a}} \quad \text { için } H(\tilde{a}) \in[0,1]
\end{aligned}
$$

Tanım 3: $\quad \tilde{a}_{i}=\left(u_{\tilde{a}_{i}}, v_{\tilde{a}_{i}}\right)(\mathrm{i}=1,2, \ldots, \mathrm{n})$ bir BS sayı olsun, öyleyse bu değerlerin birleştirilmiş hali, sezgisel bulanık ağırlıklı ortalama (SBAO) operatörü kullanılarak elde edildiğinde, yine bir SB sayı elde edilir (Xu, 2007; Yu \& Xu, 2013):

$$
\operatorname{SBAO}\left(\tilde{a}_{1}, \tilde{a}_{2}, \ldots, \tilde{a}_{n}\right)=\left(1-\prod_{j=1}^{n}\left(1-u_{\tilde{a}_{j}}\right)^{w_{j}}, \prod_{j=1}^{n} v_{\tilde{a}_{j}}{ }^{w_{j}}\right)
$$


burada $\mathrm{w}=\left\{\mathrm{w}_{1}, \mathrm{w}_{2}, \ldots, \mathrm{w}_{n}\right\} ; \mathrm{w}_{j} \in[0,1]$ ve $\sum_{\mathrm{j}=1}^{\mathrm{n}} \mathrm{w}_{\mathrm{j}}=1$ koşullarını sağlayarak $\tilde{a}_{i}(\mathrm{i}=1,2, \ldots, \mathrm{n})$ değerlerinin ağırlık vektörünü ifade eder.

Dairesel sezgisel bulanık küme (D-SBK), 2020 yılında Atanassov (2020) tarafından literatüre tanıtılmıştır. Bu yeni bulanık küme, SBK'nin uzantısıdır. Üye olma ve üye olmama derecelerinden oluşan bir sayı çemberi içermesiyle SBK'den farklıdır (Kahraman \& Alkan, 2021).

Tanım 4: $E$ sabit bir evren olsun ve E'de bulunan bir D-BS sayı $C_{r}$, x ile gösterilir ki; $C_{r}=\left\{\left\langle\mathrm{x}: u_{\mathrm{C}}(x), v_{\mathrm{C}}(x) ; \mathrm{r}\right\rangle, \mathrm{x} \in E\right\}$ bir dairesel sezgisel bulanık küme (D-SBK) olan bir nesnenin şeklidir. Burada " $u, v: \mathrm{E} \rightarrow[0,1]$ ", aşağıdaki koşullar altında sırası ile eleman $x \in E$ 'in üyelik fonksiyonunu ve üyelik-olmama fonksiyonunu D-SBK kümesine tanımlar:

$$
0 \leq u_{\mathrm{C}}(x)+v_{\mathrm{C}}(x) \leq 1 \quad \text { ve } \mathrm{r} \in[0, \sqrt{2}]
$$

Denklem 5'teki $\mathrm{r}$, her bir $\mathrm{x} \in \mathrm{E}$ elemanının etrafındaki dairenin yarıçapıdır (Kahraman \& Alkan, 2021). Belirsizlik fonksiyonu ise şu şekilde tanımlanır: $\pi_{\mathrm{C}}(x)=1-u_{\mathrm{C}}(x)-$ $v_{\mathrm{C}}(x)$. Böylece, $\mathrm{r}=0$ iken, bir D-SB sayı standard SB sayıya indirgenir.

Tanım 5: $\left\{<m_{\mathrm{i}, 1}, n_{\mathrm{i}, 1}>,<m_{\mathrm{i}, 2}, n_{\mathrm{i}, 2}>, \ldots\right\}$ SB çiftlerinden oluşan bir küme olsun. D-SB sayı olan $C_{\mathrm{i}}$ 'nin hesaplanmasında $\mathrm{i}$ (SB küme sayısı) ve $k_{i}$ (her bir kümesindeki SB çiftlerinin sayıs1) bilgilerinden yararlanılır. Setin aritmetik ortalaması aşağıdaki gibidir:

$$
C_{\mathrm{i}}=<u_{\mathrm{C}}\left(C_{\mathrm{i}}\right), v_{\mathrm{C}}\left(C_{\mathrm{i}}\right)>=<\frac{\sum_{j=1}^{k_{i}} m_{\mathrm{i}, \mathrm{j}}}{k_{i}}, \frac{\sum_{j=1}^{k_{i}} n_{\mathrm{i}, \mathrm{j}}}{k_{i}}>
$$

$C_{\mathrm{i}}$ 'nin $r_{\mathrm{i}}$ yarıçapı, Öklid mesafelerinin maksimumu ile aşağıdaki gibi elde edilir:

$$
r_{\mathrm{i}}=\max _{1 \leq j \leq k_{i}} \sqrt{\left(u_{\mathrm{C}}\left(C_{\mathrm{i}}\right)-m_{\mathrm{i}, \mathrm{j}}\right)^{2}+\left(v_{\mathrm{C}}\left(x C_{\mathrm{i}}\right)-n_{\mathrm{i}, \mathrm{j}}\right)^{2}}
$$

Tanım 6: $L^{*}=\{\langle a, b\rangle \mid a, b \in[0,1] \& a+b \leq 1\}$ olsun. Böylece, $\quad C_{r}, \quad$ " $O_{r}\left(u_{\mathrm{C}}(x), v_{\mathrm{C}}(x)\right)=\{\langle a, b\rangle \mid a, b \in[0,1]$, $\left.\sqrt{\left(u_{\mathrm{C}}(x)-a\right)^{2}+\left(v_{\mathrm{C}}(x)-b\right)^{2}} \leq r, a+b \leq 1\right\}$ " koşullarını sağlayan ve daireyi temsil eden bir fonksiyon $\left.C_{r}{ }^{*}=\left\{\left\langle\mathrm{x}: O_{r}\left(u_{\mathrm{C}}(x), v_{\mathrm{C}}(x)\right)\right)\right\rangle, \quad \mathrm{x} \in E\right\} \quad$ formunda tekrar yazılabilir. D-SBK'ler Şekil 1'de gösterilmektedir.

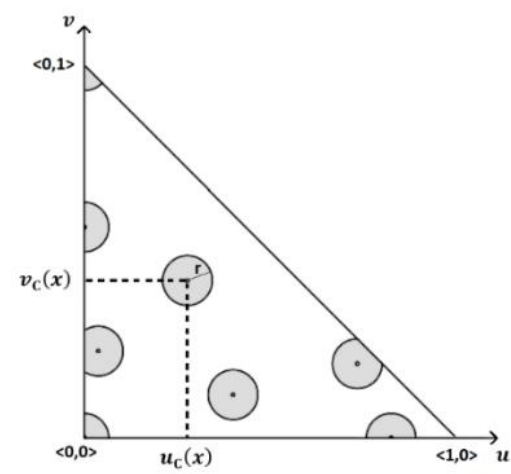

Şekil 1. Dairesel sezgisel bulanık sayıların geometrik gösterimi

Tanım 7: Burada, D-SBK'leri durulaştırmak için literatürde yeni olan D-BKS puan ve doğruluk fonksiyonları tanımlanmıştır. $\mathrm{c}=\left(u_{\mathrm{c}}, v_{c} ; r\right)$ bir D-SB say1 olsun, karar vericinin (veya e-ISSN: 2148-2683 yöneticinin) öncelik bilgisi $\lambda \in[0,1]$ ile birlikte c'nin puan fonksiyonu $S_{\mathrm{D}-\mathrm{SBK}}$ ve doğruluk fonksiyonu $H_{\mathrm{D}-\mathrm{SBK}}$ aşağıdaki gibi tanımlanır:

$$
\begin{array}{cc}
S_{\mathrm{D}-\mathrm{SBK}}(c)=\frac{u_{c}-v_{\mathrm{c}}+\sqrt{2} r(2 \lambda-1)}{3} 3 & \text { için } S_{\mathrm{D}-\mathrm{SBK}}(c) \in[-1,1] \\
H_{\mathrm{D}-\mathrm{SBK}}(\mathrm{c})=u_{c}+v_{\mathrm{c}} & \text { için } H_{\mathrm{D}-\mathrm{SBK}}(\mathrm{c}) \in[0,1]
\end{array}
$$

$\lambda$ karar vericinin modele bakış açısını yansıtır. $\lambda$ sıfıra eşitse tam karamsar bakış açısını, $\lambda$ bire eşitse tam iyimser bakış açısını gösterir. Genel kabulde $\lambda \in[0,0.5)$ karamsar bir bakış açısına, $\lambda$ $\in(0.5,1]$ ise iyimser bir bakış açısına işaret eder. $\lambda=0.5$ ise karar vericinin kayıtsız tavrını yansitır.

Tanım 8: $c_{1}=\left(u_{c_{1}}, v_{c_{1}} ; r\right)$ ve $c_{2}=\left(u_{c_{2}}, v_{c_{2}} ; r\right)$ iki D-SB sayı olsun. Böylece, sıralama kuralı şu şekilde tanımlanır:

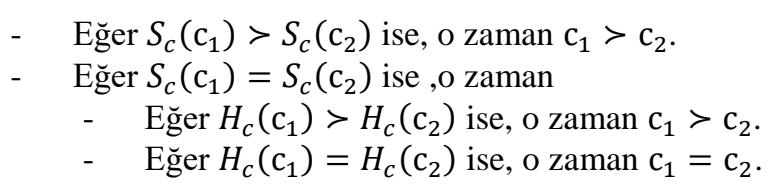

\section{Metodoloji}

$\mathrm{Bu}$ bölüm, D-SBK üzerinde yeni durulaştırma (puan ve doğruluk) fonksiyonları önererek yeni dairesel sezgisel bulanık çok kriterli karar verme (D-SB ÇKKV) yöntemini tanıtmaktadır (Çakır vd., 2021). Metodoloji, ölçütlere ve karar vericilerin incelemelerine göre alternatiflerin sıra sırasını belirlemeyi amaçlamaktadır. Önerilen D-SBK çok kriterli karar verme metodolojisinin adımları aşağıda açıklanmıştır.

Adım 1: İlk olarak problemin tanımlanması gerekir. " $\mathrm{A}=$ $\left\{\mathrm{A}_{1}, A_{2}, \ldots, A_{m}\right\}$ " alternatifler kümesi, “ $\mathrm{C}=\left\{\mathrm{C}_{1}, C_{2}, \ldots, C_{n}\right\}$ ” ölçütler kümesi ve " $D=\left\{D_{1}, D_{2}, \ldots, D_{k}\right\}$ ” karar vericiler kümesi olsun. " $\mathrm{W}_{\mathrm{C}}=\left\{\mathrm{W}_{\mathrm{C}_{1}}, W_{C_{2}}, \ldots, W_{C_{n}}\right\}$ " kriterlerin ağırlık vektörüdür ve $\mathrm{W}_{\mathrm{C}_{i}} \geq 0$ ile $\Sigma \mathrm{W}_{\mathrm{C}_{\mathrm{i}}}=1$ koşullarını sağlar. $\mathrm{Bu}$ ağırlık vektörleri, karar vericiler tarafindan belirlenir.

Adım 2: Karar vericilerden sezgisel bulanık karar matrisleri toplanır.

Adım 3: Denklem 4 ile SBAO operatörü kullanarak toplu SB karar matrisi elde edilir.

Adım 4: Kriter ağırlıklarına göre alternatiflerin toplu kararı olan $\widetilde{K}$ matrisi, SBAO operatörünü kullanılarak Denklem 4 ile hesaplanır.

Adım 5: Birleştirilmiş SB karar matrisinden Denklem 6 ve 7 ile her bir toplu karar $\widetilde{K}$ matrisi için maksimum yarıçap uzunlukları hesaplanır ve toplu kararı matrisi $\widetilde{K}$ yarıçap (D-SBK) ile revize edilir.

Adım 6: Vaka ile ilgili $\lambda$ değeri karamsar veya iyimser bir bakış açısıyla belirlenir.

Adım 7: Denklem 8 ile puan değerleri hesaplanır (durulaştırılır) ve alternatifler sıralanır.

Adım 8: Vaka için tam iyimser $(\lambda=1)$ ve tam kötümser $(\lambda=0)$ senaryosu değerlendirilir. Her $\lambda \in[0,1]$ değeri için problem çözülerek duyarlılık analizi gerçekleştirilir.

\section{Vaka Çalıșması}


Sağl1k turizmi, günümüzün uluslararası seyahat ve turizm endüstrisinde önemli bir konu haline gelmiştir (Han vd., 2018). Türkiye'ye sağlı turizmi için gelen kişi sayısı 2015 yılında 360.180 iken bu sayı 2019 yılında 662.087 kişiye yükselmiştir (USHAŞ, 2021). Yine de, dünya pazarında sağlık turizmindeki payı arttırmak gereklidir. Bu amaçla, Türkiye'nin sağlık turizmini tanıtma stratejisinde ön planda yer alacak merkezi bulmak istenmektedir. Bu amaçla, iki farklı coğrafi bölgede yer alan dört sağlık turizmi merkezi belirlenmiştir. Alternatifler $\mathrm{A} 1, A 2, A 3$ ve $A 4$ olarak adlandırılmıştır. Bunların değerlendirilmesiyle en iyi alternatif sağlık turizm destinasyonu seçilecektir. Elde edilecek sıralama ile en iyi destinasyonun merkezde yer aldığı bir pazarlama stratejisi hazırlanacaktır.

Değerlendirme yöntemi olarak önerilen dairesel sezgisel bulanık sayılar ile çok kriterli karar verme (D-SB ÇKKV) metodolojisi ele alınmıştır. Çözüm için metodolojinin adımları sirasıyla takip edilir:

Adım 1: Değerlendirme kriterleri belirlenir. Bunun için, turizm sektörünün içerisinde yer alan üç karar verici uzmanın görüşlerine başvurulmuştur. Bu karar vericiler $K V_{1}, K V_{2}$ ve $K V_{3}$ olarak adlandırılmıştır. Kriterlerin adları ve kabul edilen ağırlıkları Tablo 1'de yer almaktadır.

Tablo 1. Değerlendirme kriterleri

\begin{tabular}{|l|c|c|}
\hline $\begin{array}{c}\text { Kriter } \\
\text { kodu }\end{array}$ & Kriter adı & Ăğırlık \\
\hline$C_{1}$ & Ekipman kalitesi & 0.24 \\
\hline$C_{2}$ & İnsan kaynakları & 0.26 \\
\hline$C_{3}$ & İklim koşulları & 0.29 \\
\hline$C_{4}$ & Ulaşım imkanları & 0.21 \\
\hline
\end{tabular}

Adım 2: Eşit ağırlıklı karar vericiler (KV), alternatif merkezleri SB ayıları kullanarak ölçütlere göre değerlendirir.

$$
\begin{aligned}
& K V 1=A_{2} A_{3}\left[\begin{array}{cccc}
C_{1} & C_{2} & C_{3} & C_{4} \\
A_{3} & A_{4}
\end{array}\left[\begin{array}{cccc}
\langle 0.6,0.2\rangle & \langle 0.4,0.3\rangle & \langle 0.5,0.1\rangle & \langle 0.3,0.4\rangle \\
\langle 0.5,0.1\rangle & \langle 0.5,0.4\rangle & \langle 0.2,0.4\rangle & \langle 0.6,0.2\rangle \\
\langle 0.8,0.2\rangle & \langle 0.3,0.5\rangle & \langle 0.2,0.4\rangle & \langle 0.3,0.5\rangle \\
\langle 0.1\rangle & \langle 0.6,0.1\rangle & \langle 0.5,0.2\rangle & \langle 0.4,0.3\rangle
\end{array}\right]\right.
\end{aligned}
$$

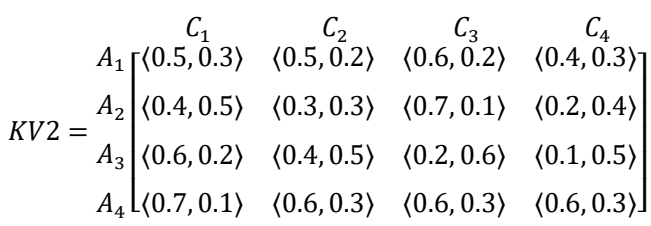

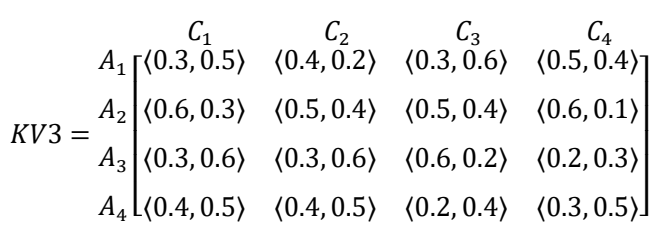

Adım 3: SBAO operatörü tarafindan üç sezgisel bulanık karar matrisinin birleştirilmiş SB karar matrisi aşağıdaki gibidir:

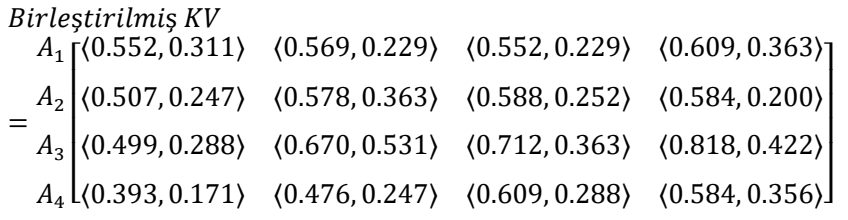

Adım 4: Alternatiflerin birleştirilmiş kararları, Adım 1'de belirtilen ölçüt ağırlıklarına göre SBAO operatörü tarafından elde edilir.

$$
\widetilde{K}=A_{2}\left[\begin{array}{c}
A_{1} \\
A_{3} \\
A_{4}
\end{array}\left[\begin{array}{c}
\langle 0.5688,0.2715\rangle \\
\langle 0.6904,0.3915\rangle \\
\langle 0.5247,0.2553\rangle
\end{array}\right]\right.
$$

Adım 5: Her bir birleştirilmiş karar için, maksimum yarıçap uzunlukları, Adım 3 'teki birleştirilmiş sezgisel bulanık karar matrisi yardımıyla Denklem 6 ve Denklem 7 ile aşağıdaki gibi hesaplanır:

$$
\widetilde{K}=A_{2}\left[\begin{array}{c}
A_{1} \\
A_{3} \\
A_{4}
\end{array}\left[\begin{array}{c}
\langle 0.5688,0.2715 ; 0.209\rangle \\
\langle 0.6964,0.2627 ; 0.130\rangle \\
\langle 0.5247,0.2553 ; 0.145\rangle
\end{array}\right]\right.
$$

Adım 6: Bu vaka için yönetim iyimser bakış açısını $\lambda=0.7$ olarak belirlemek istemektedir.

Adım 7: Denklem 8'de önerilen puan fonksiyonu (durulaştırma fonksiyonu) yardımıyla alternatiflerin puanları şu şekilde bulunmuştur: $A_{1}=0.265, A_{2}=0.248, A_{3}=0.300$, $A_{4}=0.236$

Böylece, alternatiflerin siralaması $A_{3}>A_{1}>A_{2}>A_{4}$ olarak ortaya çıkmıştır.

Adım 8: Her $\lambda \in[0,1]$ değeri için değerler ve sıralama sonuçları Tablo 2'de verilmiştir.

Tablo 2. $\lambda$ değeri için duyarlılık analizi

\begin{tabular}{|l|c|c|c|c|c|}
\hline \multicolumn{1}{|c|}{ Score } & $\boldsymbol{A}_{\mathbf{1}}$ & $\boldsymbol{A}_{\mathbf{2}}$ & $\boldsymbol{A}_{\mathbf{3}}$ & $\boldsymbol{A}_{\mathbf{4}}$ & Siralama \\
\hline$\lambda=0$ & 0.001 & 0.040 & 0.054 & 0.021 & $A_{3}>A_{2}>A_{4}>A_{1}$ \\
\hline$\lambda=0.1$ & 0.038 & 0.070 & 0.089 & 0.052 & $A_{3}>A_{2}>A_{4}>A_{1}$ \\
\hline$\lambda=0.2$ & 0.076 & 0.099 & 0.125 & 0.083 & $A_{3}>A_{2}>A_{4}>A_{1}$ \\
\hline$\lambda=0.3$ & 0.114 & 0.129 & 0.160 & 0.113 & $A_{3}>A_{2}>A_{1}>A_{4}$ \\
\hline$\lambda=0.4$ & 0.152 & 0.159 & 0.195 & 0.144 & $A_{3}>A_{2}>A_{1}>A_{4}$ \\
\hline$\lambda=0.5$ & 0.190 & 0.189 & 0.230 & 0.175 & $A_{3}>A_{1}>A_{2}>A_{4}$ \\
\hline$\lambda=0.6$ & 0.227 & 0.219 & 0.265 & 0.206 & $A_{3}>A_{1}>A_{2}>A_{4}$ \\
\hline$\lambda=0.7$ & 0.265 & 0.248 & 0.300 & 0.236 & $A_{3}>A_{1}>A_{2}>A_{4}$ \\
\hline$\lambda=0.8$ & 0.303 & 0.278 & 0.336 & 0.267 & $A_{3}>A_{1}>A_{2}>A_{4}$ \\
\hline$\lambda=0.9$ & 0.341 & 0.308 & 0.371 & 0.298 & $A_{3}>A_{1}>A_{2}>A_{4}$ \\
\hline$\lambda=1$ & 0.379 & 0.338 & 0.406 & 0.328 & $A_{3}>A_{1}>A_{2}>A_{4}$ \\
\hline
\end{tabular}

Tam kötümser durum $\lambda=0$ olduğunda ortaya çıkar ve sıralama sonucu $A_{3}>A_{2}>A_{4}>A_{1}{ }^{\prime}$ dir. Tam iyimser durum $\lambda=1$ olduğunda ortaya çıkar ve siralama sonucu $A_{3}>A_{1}>A_{2}>A_{4}$ olur. Kötümserden iyimsere doğru diziler incelendiğinde, en kötü alternatif $A_{1}$ 'den $A_{4}$ 'e değişir, ancak en iyi alternatif $A_{3}$ değiş̧mez. İyimser senaryoya doğru, $A_{1}$ alternatifi siralamada ileriye giderken, alternatif $A_{4}$ siralamada gerilemeye başlar. Böylece yönetici tarafından verilen $\lambda$ değerinin puana ve sıralamaya yansımasından bahsedilebilir.
$C_{1}$

$C_{2}$

$C_{3}$

$C_{4}$

\section{Sonuç}


Çok kriterli karar verme yaklaşımları bulanık mantık ile bütünleştirilerek uygulanarak insan doğasındaki belirsizlikleri modellere aktarmada sıklıkla kullanılmaktadır. Bu çalışma ile alternatifleri belirlenen ölçütlere dayalı olarak değerlendirmek için dairesel bulanık çok kriterli karar verme üzerine yeni bir metodoloji önerilmektedir. Sezgisel bulanıklığın yeni bir uzantısı olan D-SBK'yi sıralamak için yeni durulaştırma fonksiyonları önerilmiştir. Yöntem, sağlık turizmi merkezi seçiminde uygulanmıştır. Duyarlılık analizi ile birlikte $\lambda$ parametresindeki değişkenliğin etkisi araştırılmıştır.

Gelecekteki araştırmalar için karar verme sürecinde yeni durulaştırma ve doğruluk fonksiyonları tanımlanabilir. Çok kriterli karar verme metodolojisi, yeni operatörler uyarlanarak zenginleştirilmelidir. Gerçek hayattaki vakalar, dairesel sezgisel çok kriterli karar verme prosedürleri ile uygulanmalıdır. Sağlık turizmi merkezi problemlerinde ele alınan kriter ve alternatif sayıları arttırılarak daha geniş kapsamlı değerlendirme modelleri oluşturulabilir. Ayrıca, Türkiye'yi ele alan çalışmanın diğer ülkelerde yapılan çalışmaların sonuçları ile karşılaştırılarak rekabet analizi yapılabilir.

\section{Kaynakça}

Akinboade, O. A., \& Braimoh, L. A. (2010). International tourism and economic development in South Africa: A Granger causality test. International Journal of Tourism Research, 12(2), 149-163. doi: 10.1002/jtr.743

Amouzagar, S., Mojaradi, Z., Izanloo, A., Beikzadeh, S., \& Milani, M. (2016). Qualitative examination of health tourism and its challenges. International Journal of Travel Medicine and Global Health, 4(3), 88-91. doi: 10.21859/IJTMGH040304

Atanassov, K. T. (1986). Intuitionistic fuzzy sets. Fuzzy Sets and Systems, 20(1), 87-96. doi: 10.1016/S0165-0114(86)80034-3

Atanassov, K. T. (2020). Circular intuitionistic fuzzy sets. Journal of Intelligent \& Fuzzy Systems, 39(5), 5981-5986. doi: 10.3233/JIFS-189072

Carrera, P. M., \& Bridges, J. F. (2006). Globalization and healthcare: understanding health and medical tourism. Expert review of pharmacoeconomics \& outcomes research, 6(4), 447-454. doi: 10.1586/14737167.6.4.447

Chen, S. M., \& Tan, J. M. (1994). Handling multicriteria fuzzy decision-making problems based on vague set theory. Fuzzy sets and systems, 67(2), 163-172. doi: 10.1016/01650114(94)90084-1

Çakır, E., Taş, M.A. \& Ulukan, Z. (2021). Circular Intuitionistic Fuzzy Sets in Multi Criteria Decision Making. 11th International Conference On Theory And Application Of Soft Computing, Computing With Words, Perception And Artificial Intelligence (ICSCCW 2021). Antalya, Turkey. 23 - 24 August 2021.

Çakır, E., \& Ulukan, Z. (2020). Sustainable health tourism site selection with a fuzzy linguistic prolog. In Developments of Artificial Intelligence Technologies in Computation and Robotics: Proceedings of the 14th International FLINS Conference (FLINS 2020), pp. 1456-1463.
Ghasemi, P., Mehdiabadi, A., Spulbar, C., \& Birau, R. (2021). Ranking of Sustainable Medical Tourism Destinations in Iran: An Integrated Approach Using Fuzzy SWARAPROMETHEE. Sustainability, 13(2), $683 . \quad$ doi: 10.3390/su13020683

Han, J. S., Lee, T. J., \& Ryu, K. (2018). The promotion of health tourism products for domestic tourists. International Journal of Tourism Research, 20(2), 137-146. doi: 10.1002/jtr.2161

Hung, Y. H., Ma, J., Chou, J. C. L., \& Tzeng, G. H. (2014, September). Improving medical tourism services based on a hybrid MCDM model combined by DANP and VIKOR techniques. In 2014 IEEE International Conference on Management of Innovation and Technology (pp. 486-488). IEEE. doi: 10.1109/ICMIT.2014.6942475

Izadi, M., Ayoobian, A., Nasiri, T., Joneidi, N., Fazel, M., \& Hosseinpourfard, M. J. (2012). Situation of health tourism in Iran; opportunity or threat. Journal of Military Medicine, 14(2), 69-75.

Kahraman, C., \& Alkan, N. (2021). Circular intuitionistic fuzzy TOPSIS method with vague membership functions: Supplier selection application context. Notes on Intuitionistic Fuzzy Set, 27(1), 24-52. doi: 10.7546/nifs.2021.27.1.24-52

Kantar, G., \& Işık, E. (2014). Türkiye'de sağlık turizmi. Sağlık Akademisyenleri Dergisi, 1(1), 15-20. https://dergipark.org.tr/en/download/article-file/752395

Kušen, E. (2011). Positioning medical tourism in the broader framework of health tourism. Tourism: an international interdisciplinary journal, 59(1), 95-99.

Kutlu Gündoğdu, F., \& Kahraman, C. (2019). Spherical fuzzy sets and spherical fuzzy TOPSIS method. Journal of intelligent \& fuzzy systems, 36(1), 337-352. doi: 10.3233/JIFS-181401

Lee, C. W., \& Li, C. (2019). The process of constructing a health tourism destination index. International journal of environmental research and public health, 16(22), 4579. doi: 10.3390/ijerph16224579

Pan, X., Yang, Z., Han, F., Lu, Y., \& Liu, Q. (2019). Evaluating potential areas for mountain wellness tourism: A case study of Ili, Xinjiang Province. Sustainability, 11(20), 5668. doi: $10.3390 /$ su11205668

Roy, J., Chatterjee, K., Bandyopadhyay, A., \& Kar, S. (2018). Evaluation and selection of medical tourism sites: A rough analytic hierarchy process based multi-attributive border approximation area comparison approach. Expert Systems, 35(1), e12232. doi: 10.1111/exsy.12232

Sariisik, M., Turkay, O., \& Akova, O. (2011). How to manage yacht tourism in Turkey: A swot analysis and related strategies. Procedia-Social and Behavioral Sciences, 24, 1014-1025. doi: 10.1016/j.sbspro.2011.09.041

Smarandache, F. (1998). Neutrosophy: neutrosophic probability, set, and logic: analytic synthesis \& synthetic analysis.

T. C. Kültür ve Turizm Bakanlığı. (2021). Türkiye'de Sağllk Turizmine Yönelik Yapılan Çalışmaları ve Etkinlikler. https://yigm.ktb.gov.tr/TR-11479/turkiye39de-saglikturizmine-yonelik-yapilan-calismalar-.html. Erişim Tarihi: 14.07.2021 
Torra, V. (2010). Hesitant fuzzy sets. International Journal of Intelligent Systems, 25(6), 529-539. doi: 10.1002/int.20418

Tureac, C. E., \& Turtureanu, A. (2010). Types and forms of tourism. Acta Universitatis Danubius. Economica, 4(1). http://www.journals.univdanubius.ro/index.php/oeconomica/article/view/60/56

Türkiye Seyahat Acentaları Birliği (TÜRSAB). (2021). Turizm gelirleri. https://www.tursab.org.tr/istatistikler-icerik/turizmgeliri. Erişim Tarihi: 10.07.2021

Uluslararası Sağlık Hizmetleri A. Ş. (USHAŞ). (2021). Sağlık Turizmi Verileri. https://www.ushas.com.tr/saglik-turizmiverileri/Erişim Tarihi: 10.07.2021

World Tourism Organization (UNWTO). (2018). Exploring Health Tourism - Executive Summary. https://www.eunwto.org/doi/pdf/10.18111/9789284420308 Erişim Tarihi: 11.07.2021

World Tourism Organization (UNWTO). (2019). International Tourism Highlights, 2019 Edition, Madrid. doi: 10.18111/9789284421152 Erişim Tarihi: 11.07.2021

$\mathrm{Xu}, \mathrm{Z}$. (2007). Intuitionistic fuzzy aggregation operators. IEEE Transactions on fuzzy systems, 15(6), 1179-1187. doi: 10.1109/TFUZZ.2006.890678

Yager, R. R. (2013, June). Pythagorean fuzzy subsets. In 2013 joint IFSA world congress and NAFIPS annual meeting (IFSA/NAFIPS) (pp. 57-61). IEEE. doi: 10.1109/IFSANAFIPS.2013.6608375

Yang, J. Y., Paek, S., Kim, T. T., \& Lee, T. H. (2015). Health tourism: Needs for healing experience and intentions for transformation in wellness resorts in Korea. International Journal of Contemporary Hospitality Management, 27(8), 1881-1904. doi: 10.1108/IJCHM-11-2013-0505

Yu, X., \& Xu, Z. (2013). Prioritized intuitionistic fuzzy aggregation operators. Information Fusion, 14(1), 108-116. doi: 10.1016/j.inffus.2012.01.011

Zadeh, L. A. (1965). Fuzzy Sets. Information and Control, 8(3), 338-353. doi: 10.1016/S0019-9958(65)90241-X 